\title{
Nephrogenic Ascites Uncommon Cause of Exsudative Ascites
}

\author{
Dr. Lairani ${ }^{1 *}$, Dr. Jiddi ${ }^{1}$, Dr. Aboutarik ${ }^{1}$, Pr Ait Errami ${ }^{1}$, Pr Samlani ${ }^{1}$, Pr Oubaha ${ }^{2}$, Pr Krati ${ }^{1}$
}

\footnotetext{
${ }^{1}$ Department of Gastroenterology at Mohamed VI Universal Hospital of Marrakesh, Morocco

${ }^{2}$ Department of Physiology at Faculty of Medicine and Pharmacy of Marrakesh, Morocco
}

DOI: $10.36347 /$ simcr.2020.v08i02.026

| Received: 13.02.2020 | Accepted: 20.02.2020 | Published: 22.02.2020

*Corresponding author: Fatima Ezzahra Lairani

Abstract

Nephnogenlc ascites is a rare condition characterized by the presence of massive ascites in patients with end stage renal disease (ESRD). Although the pathogenesis remains poorly understood, several factors have been considered. Nephrogenic ascites is associated with a grave prognosis, especially if treatment is not institued. Treatment options are limited, of those kidney transplant seems to be the most effective, however, special attention should be paid to intensive hemodialysis. Through this observation, we study clinicopathological and therapeutic features of this pathology. We report one case of a 64 years old patient with a past medical history of arterial hypertension, followed for a chronic renal failure undergoing hemodialysis for two years. Diagnostic paracentesis was done and found to be exudative. After detailed workup, hepatic, cardiac, infectious and malignant causes for ascites were ruled out. The diagnosis of ascites of nephrogenic origin was made. The patient has been treated conservatively with salt/fluid restriction and intensive hemodialysis with ultrafiltration which was very successful in our case.

Keywords: Nephnogenlc ascites, end stage renal disease (ESRD), kidney transplant, hemodialysis.

Copyright @ 2020: This is an open-access article distributed under the terms of the Creative Commons Attribution license which permits unrestricted use, distribution, and reproduction in any medium for non-commercial use (NonCommercial, or CC-BY-NC) provided the original author and source are credited.

\section{INTRODUCTION}

Nephrogenic ascites refers to refractory ascites of unknown etiology occuring in patients with ESRD, many of whom are undergoing hemodialysis [1]. Its pathogenesis remains unkown. Contributing mechanism of nephrogenic acites may include inadequate dialysis and ultrafiltration, poor nutrition and increased peritoneal membrane permeability in combination with impaired peritoneal lymphatic reabsorption [2]. It has limited treatment options and is associated with poor prognosis [3].

We report in this document the case of a 64 years old woman who presented with ascites of nephrogenic origin and review this entity.

\section{Case Presentation}

A 64 years old patient with end stage renal disease secondary to hypertensive nephropathy on once weekly maintenance hemodialysis since 2 years (non compliance to the twice weekly hemodialysis prescribed due to financial problems), was admitted to our department with progressively worsening abdominal distension of 3 months duration, associated with fatigue, anorexia and weight loss, without abdominal pain. She had no notable medical history except for arterial hypertension for 10 years.

Physical examination revealed a cachectic woman $(B M I=15 \mathrm{~kg} / \mathrm{m} 2)$, mild peripheral edema, a markedly distended abdomen with normoactive bowel sounds, massive ascites and mild tenderness. No icterus, palpable organomegaly, nor lymphadenopathy were noted. The remainder of the physical examination was unremarkable.

Her laboratory investigations revealed hemoglobin $(\mathrm{Hb})$ of $7.3 \mathrm{~g} / \mathrm{dL}$, a white blood cell count of 6390, a normal platelet count, blood urea of $1.78 \mathrm{~g} / \mathrm{L}$, serum creatinine of $83 \mathrm{mg} / \mathrm{L}$, total protein level of 63 $\mathrm{g} / \mathrm{L}$, an albumin level of $33 \mathrm{~g} / \mathrm{L}$. Coagulation studies, thyroid function tests, and serum sodium, potassium, chloride, and bicarbonate concentrations were all normal.

A diagnostic / therapeutic paracentesis was performed with the removal of $3 \mathrm{~L}$ of clear yellow fluid. Ascitic fluid analysis revealed protein of $51.1 \mathrm{~g} / \mathrm{L}$. The ascitic fluid white cell count was 240 cells $/ \mathrm{mm} 3$, with $60 \%$ cells being polynuclear neutrophils. Ascitic fluid adenosine deaminase was low at 14UI/L. Cultures of fluid were negative for bacterial elements including 
Mycobacterium Tuberculosis. Cytology was also negative for malignant cells.

An abdominal computed tomography showed ascites, good portal vein flow, no masses, and normal liver size with a homogenous consistency. Gastroscopy and colonoscopy were without any particularities as well. Echocardiography showed a preserved cardiac function. antinuclear and anti-dsDNA antibodies were not detected.

A diagnostic laparoscopy was performed to assess the peritoneum for infectious or malignant disease, liver surface morpholgy, and presence of portal hypertensive collateral vessels. No gross abnormalities were observed : no signs of peritoneal carcinomatosis or tuberculosis, no signs of cirrhosis, and no metastatic lymph nodes. Systematic peritoneal biopsies showed discrete inflammation with no malignant cells nor granulomas.

The patient was then diagnosed to have nephrogenic ascites and underwent aggressive sessions of thrice weekly hemodialysis. This was accompanied by dietary salt restriction and oral nutritional protein supplementation. Therapeutic large volume paracentesis was also performed once every 3 to 4 weeks with intravenous albumin infusions to relieve her of abdominal discomfort. A gradual reduction in ascites was noted which eventually resolved after 4 months of follow up.

\section{DisCUSSION}

This case brings to light the condition termed as nephrogenic ascites, a problem that has been most often described in ESRD patients on hemodialysis.

Nephrogenic ascites has been known by several names such as nephrogenous ascites, hemodialysis associated ascites, dialysis ascites, idiopathic ascites, or ascites associated with ESRD [4].

It was first reported in the early 1970 s in patients with ESRD [1]. It is most often seen in patients on hemodialysis, but has been described earlier in the course of renal failure occasionally [2].

Nephrogenic ascites could be possibly due to a combination of factors such as poor nutrition, late presentation leading to delay in initiation of appropriate renal replacement therapy and inadequate dialysis. When dialysis is not being covered by the state or insurance policies, this necessitates patients to fund their own treatments, leading to poor compliance with the dialysis schedule [2], which was unfortunately the case for our patient.

The pathogenesis of «dialysis ascites» is not well understood and has been a subject of much debate. The formation of ascites is a dynamic process dependant upon factors influencing the formation and resorption of ascitic fluid [1].

Several pathogenetic factors including elevated hepatic vein hydrostatic pressure, fluid retention, increased peritoneal membrane permeability, and impaired lymphatic drainage have been considered [4] Early reports incriminated prior peritoneal dialysis which may play a role either altering peritoneal membrane permeability or inducing peritoneal inflammation. However, the low incidence of prior peritoneal dialysis in patients with nephrogenic ascites makes its role very unlikely in the pathogenesis [1]. Although other authors have suggested an etiologic role for congestive heart failure in patients with nephrogenic ascites, the contribution is unclear, and need further investigations [1].

To a certain extent, the nephrogenic ascites is a diagnosis of exclusion. Before the diagnosis of nephrogenic ascites is established, other causes of peritoneal fluid accumulation such as cirrhosis, abdominal malignancy, pericardial constriction or tamponade, inferior vena caval obstruction, tuberculous peritonitis, and pancreatitis must be ruled out [5].

The presentation of dialysis ascites is variable, there being no truly characteristic clinical or laboratory findings. Some patients develop swelling of the abdomen only, whereas others manifest signs of fluid excess elsewhere in the body; in the form of pleural effusion or peripheral edema [6].

The ascitic fluid itself is typically high in protein content, suggesting an exudative process. The peritoneal fluid cell count, however, is normal. Microscopy of the peritoneal membrane is usually unremarkable [6] but more often shows chronic inflammation and mesothelial cell proliferation with variable degree of submesothelial fibrosis [2].

Nephrogenic ascites is usually associated with a grave prognosis. The average survival ranges from seven to 10.7 months, with $44 \%$ of patients dying within 15 months of diagnosis [2]. The clinical course in a patient with refractory nephrogenic ascites is marked by worsening cachexia and progression to death [7]. In contrast, the majority of responders and longterm survivors had resolution of ascites with treatment. The survival rate in those patients is found to be closer to the survival rate of ESRD without ascites [7].

Management of nephrogenic ascites is complex and includes a combination of intensive dialysis with good ultrafiltration, intradialytic albumin infusions along with a highprotein diet which is given in the intial stages to control the ascites [2]. Paracentesis may acutely relieve abdominal swelling, but, if repeated, paracentesis may lead to protein 
depletion and infection, without lasting regression of the ascites [6].

Daily hemodialysis was effective in controlling ascites in about $35 \%$ to $78 \%$ of patients in the literature. The reason why many physicians fail to remove sufficient fluid from patients with nephrogenic ascites is probably that those patients are particularly prone to developing hypotension during ultrafiltration. When this happens, ultrafiltration is discontinued and intraveinous fluid may be required to maintain blood pressure. This results in inadequate removal of excess fluid, and control of ascites is not achieved [5]. When successful, daily hemodialysis results in resolution of ascites within 1-2 weeks [1].

Continuous ambulatory peritoneal dialysis (CAPD) and peritoneovenous shunts (PVS) are the other treatment options [2].

CAPD may be effective in the treatment of ascites by controlling ascites formation. This is by reducing the intraperitoneal fluid protein concentration thus limiting osmotic fluid shift into the peritoneal space. Within six months of continued treatment, the amount of total protein excretion in the dialysis effluent decreases over time, with a subsequent rise in serum protein and albumin levels and resolution of ascites [2].

PVS have been reported in the surgical literature to be effective in controlling nephrogenic ascites in conjunction with continued hemodialysis. Long term success was dependent on mainting shunt patency [1]. Complications of shunt placement are seen in about half of the cases, and include catheter tip migration, infection and occlusion [2].

Renal transplant is considered the definitive treatment for nephrogenic ascites and is associated with complete resolution of ascites within 2 to 6 weeks along as improvement in quality of life [1]. However, the recurrence of ascites may occur at any time of graft failure or at any time up to 3 years due to various reasons [7].
Our patient was on a once-weekly dialysis and was clearly underdialyzed which resulted on the formation of ascites. Intensification of the dialysis regimen resulted in a clear objective improvement including a complete resolution of ascites and improvement of quality of life.

\section{CONCLUSION}

Nephrogenic ascites is a rare condition occuring in patients with ESRD associated with poor prognosis and unknown but probably multifactorial causes. Intensive dialysis should be the initial therapy as it offers a better hope for recovery and improvement in the quality of life. CAPD and PVS may be considered as alternative treatments, however renal transplantation seems to be the only effective therapeutic approach.

\section{REFERENCES}

1. Han SH, Reynolds TB, Fong TL. Nephrogenic ascites. Analysis of 16 cases and review of the literature. Medicine. 1998 Jul;77(4):233-45.

2. Nayak-Rao S. Nephrogenic ascites-Still an intractable problem?. Saudi Journal of Kidney Diseases and Transplantation. 2015 Jul $1 ; 26(4): 773$.

3. Cintin C, Joffe P. Nephrogenic ascites: case report and review of the literature. Scandinavian journal of urology and nephrology. 1994 Sep 1;28(3):3114.

4. Hammond TC, Takiyyuddin MA. Nephrogenic ascites: a poorly understood syndrome. Journal of the American Society of Nephrology. 1994 Nov 1;5(5):1173-7.

5. Gunal AI, Karaca I, Celiker H, Ilkay E, Duman S. Strict volume control in the treatment of nephrogenic ascites. Nephrology Dialysis Transplantation. 2002 Jul 1;17(7):1248-51.

6. Popli S, Daugirdas JT, Ing TS. Dialysis ascites.1980.

7. Al-zakhari R, Alataby H, Freg G, Moussa J, Mossayebi E, Ebrahimi F. A Real Neglected Problem With a Grave Prognosis: Nephrogenic Ascites. Journal of Medical Cases. 2020 Jan 31;11(1):26-9. 